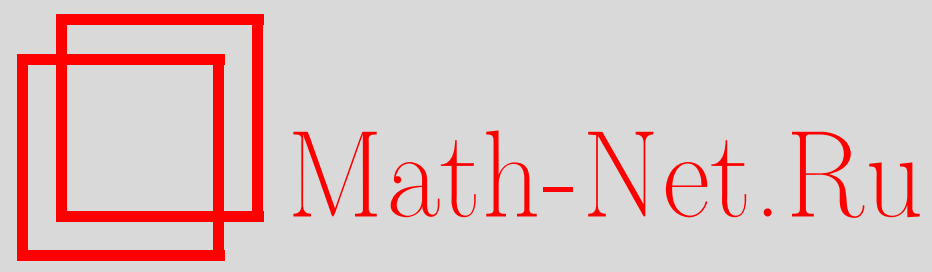

А. В. Устинов, Дискретный аналог формулы суммирования Пуассона, Матем. заметки, 2003, том 73, выпуск 1, 106112

DOI: https://doi.org/10.4213/mzm173

Использование Общероссийского математического портала Math-Net.Ru подразумевает, что вы прочитали и согласны с пользовательским соглашением http://www.mathnet.ru/rus/agreement

Параметры загрузки:

IP : 3.85 .5 .30

26 апреля 2023 г., 16:48:36

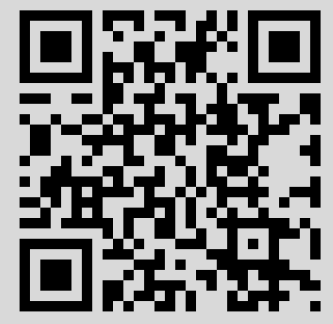




\section{ДИСКРЕТНЫЙ АНАЛОГ \\ ФОРМУЛЫ СУММИРОВАНИЯ ПУАССОНА}

\section{А. В. Устинов}

В первой части статьи доказывается дискретный аналог формулы суммирования Пуассона. Во второй части работы излагается элементарное доказательство функционального уравнения для функции $\theta(t)$, основанное на полученной формуле суммирования.

Библиографоял: 4 названия.

1. Введение. Пусть $\mathscr{S}$ - пространство бесконечно дифференцируемых функций $f$ : $\mathbb{R} \rightarrow \mathbb{C}$, убьвающих быстрее, чем любая положительная степень, т.е. таких, что для любого натурального $n$

$$
\lim _{x \rightarrow \pm \infty}|x|^{n} f(x)=0
$$

Определим преобразование Фурье $\widehat{f}$ функции $f \in \mathscr{S}$ формулой

$$
\widehat{f}(y)=\int_{-\infty}^{\infty} e^{2 \pi i x y} f(x) d x
$$

Такой интеграл сходится при всех действительных значениях $y$ и задает функцию $\widehat{f}(y) \in \mathscr{S}$.

Известно, что сумма значений функции в точках равномерной сетки связана с аналогичной суммой значений ее преобразования Фурье. Подобная связь описывается формулой суммирования Пуассона. Она допускает различные варианты записи. Для функции $f \in \mathscr{S}$ она может быть записана без остаточного члена:

$$
\sum_{m=-\infty}^{\infty} f(m)=\sum_{m=-\infty}^{\infty} \widehat{f}(m)
$$

(см. [1]).

Работа выполнена при частичной поддержке Российского фонда фундаментальных исследований, грант № 01-01-00738. 
Формула суммирования Пуассона используется в различных задачах математического анализа и теории чисел. Например, для функции

$$
\theta(t)=\sum_{n=-\infty}^{\infty} e^{-\pi t n^{2}}
$$

определенной при $t>0$, с помощью формулы (1) можно доказать функциональное уравнение

$$
\theta\left(t^{-1}\right)=\sqrt{t} \theta(t)
$$

(см. [1], [2]). Варианты формулы суммирования Пуассона с остаточным членом позволяют находить точное значение суммы Гаусса (см. [3], [2]) и получать оценки тригонометрических сумм (см. [2]). Еще несколько примеров можно найти в книге [4].

В настоящей работе рассматриваются функции, заданные в конечном числе точек равномерной сетки. Для таких функций доказывается дискретный аналог формулы (1), который связьвает сумму значений функции в узлах более редкой равномерной сетки с суммой ее конечных коэффициентов Фурье. Затем полученная формула применяется к доказательству функционального уравнения функции $\theta(t)$. При этом подход к доказательству равенства (3) является элементарным.

Аналогично дискретный аналог равенства (1) может быть использован и в других задачах, в которых применяется обычная формула суммирования Пуассона.

2. Дискретный аналог формулы суммирования Пуассона. Далее в качестве равномерной сетки будем рассматривать точки с целыми координатами, т.е. будем предполагать, что функция $f(x)$ определена для всех целых $x$ из интервала $0 \leqslant x<p$, где $p$ - некоторое натуральное число.

Известно, что в каждой из этих точек функция $f(x)$ представляется своим конечным рядом Фурье

$$
f(x)=\sum_{k=0}^{p-1} C_{p}(k) e^{2 \pi i k x / p}, \quad 0 \leqslant x<p,
$$

где $C_{p}(k)$ - конечные коэффициенты $\Phi$ урье функции $f(x)$, которые находятся по формуле

$$
C_{p}(k)=\frac{1}{p} \sum_{x=0}^{p-1} f(x) e^{-2 \pi i k x / p}, \quad 0 \leqslant k<p .
$$

Следующее утверждение можно считать дискретным аналогом формулы (1).

ТЕОРема 1. Пусть $p_{1}, p_{2}$ - натуральные числа, $p=p_{1} p_{2}$, функиия $f(x)$ определена для всех иелых $x$ из интервала $0 \leqslant x<p$ и $C_{p}(k)$-ее конечные коэффичиенты Фурье. Тогда справедливо равенство

$$
\sum_{y=0}^{p_{2}-1} f\left(p_{1} y\right)=p_{2} \sum_{n=0}^{p_{1}-1} C_{p}\left(p_{2} n\right)
$$


ДоКАЗАТЕЛЬСТво. Преобразуем первую сумму при помощи формулы (4):

$$
\sum_{y=0}^{p_{2}-1} f\left(p_{1} y\right)=\sum_{y=0}^{p_{2}-1} \sum_{k=0}^{p-1} C_{p}(k) e^{2 \pi i k p_{1} y / p}=\sum_{k=0}^{p-1} C_{p}(k) \sum_{y=0}^{p_{2}-1} e^{2 \pi i k y / p_{2}}
$$

Далее, так как

$$
\sum_{y=0}^{p_{2}-1} e^{2 \pi i k y / p_{2}}=p_{2} \delta_{p_{2}}(k)= \begin{cases}p_{2}, & \text { если } k \equiv 0\left(\bmod p_{2}\right) \\ 0, & \text { если } k \neq 0\left(\bmod p_{2}\right)\end{cases}
$$

то

$$
\sum_{y=0}^{p_{2}-1} f\left(p_{1} y\right)=\sum_{k=0}^{p-1} C_{p}(k) p_{2} \delta_{p_{2}}(k)=p_{2} \sum_{n=0}^{p_{1}-1} C_{p}\left(p_{2} n\right) .
$$

3. Доказательство функционального уравнения для функции $\theta(t)$. Доказательству равенства (3) предпошлем несколько вспомогательных утверждений.

Лемма 1. Пусть $q_{1}, q_{2}$ - натуральные числа и $q=2 q_{1} q_{2}$. Тогда справедливо равенство

$$
\frac{q_{1}}{2^{q}} \sum_{m=-q_{2}}^{q_{2}} C_{q}^{q_{1}\left(q_{2}+m\right)}=\sum_{n=0}^{q_{1}-1}\left(\cos \frac{\pi n}{q_{1}}\right)^{q} .
$$

ДокАЗАТЕЛЬСтво. Рассмотрим функцию $f(x)=C_{q}^{x}$, определенную при целых $x$ из интервала $0 \leqslant x<q$, и найдем ее конечные коэффициенты $\Phi$ урье:

$$
\begin{aligned}
C_{q}(k) & =\frac{1}{q} \sum_{x=0}^{q-1} C_{q}^{x} e^{-2 \pi i k x / q}=\frac{1}{q}\left[\left(1+e^{-2 \pi i k / q}\right)^{q}-1\right] \\
& =\frac{1}{q}\left[e^{-\pi i k}\left(e^{\pi i k / q}+e^{-\pi i k / q}\right)^{q}-1\right]=\frac{1}{q}\left[(-1)^{k}\left(2 \cos \frac{\pi k}{q}\right)^{q}-1\right] .
\end{aligned}
$$

Применяя теорему 1 к функции $f(x)=C_{q}^{x}$ с $p_{1}=q_{1}, p_{2}=2 q_{2}$ и $p=2 q_{1} q_{2}$, приходим к равенству

$$
\sum_{y=0}^{2 q_{2}-1} C_{q}^{q_{1} y}=\frac{2 q_{2}}{q} \sum_{n=0}^{q_{1}-1}\left(2 \cos \frac{\pi n}{q_{1}}\right)^{q}-1
$$

Отсюда

$$
\frac{q_{1}}{2^{q}} \sum_{y=0}^{2 q_{2}} C_{q}^{q_{1} y}=\sum_{n=0}^{q_{1}-1}\left(\cos \frac{\pi n}{q_{1}}\right)^{q},
$$

что равносильно утверждению леммы. 
Лемма 2. Пусть $t>0$ - действительное число такое, что произведение $\pi t$ рационально. Пусть далее

$$
\pi t=\frac{a}{b}, \quad(a, b)=1, \quad z \geqslant 1, \quad q_{1}=a z, \quad q_{2}=b z, \quad q=2 q_{1} q_{2} .
$$

Будем также предполагать, что величинь $M, N, m$ и п удовлетворяют неравенствам

$$
0 \leqslant M, N \leqslant \sqrt{z}, \quad|m| \leqslant M, \quad|n| \leqslant N .
$$

Тогда справедливы следующие асимптотические формуль:

$$
\begin{aligned}
\frac{q_{1}}{2^{q}} C_{q}^{q_{1}\left(q_{2}+m\right)} & =\sqrt{t} e^{-\pi t m^{2}}\left(1+O\left(\frac{M^{4}}{z^{2}}\right)\right), \\
\left(\cos \frac{\pi n}{q_{1}}\right)^{q} & =e^{-\pi n^{2} / t}\left(1+O\left(\frac{N^{4}}{z^{2}}\right)\right),
\end{aligned}
$$

где константы в знаках $O$ могут зависеть от а $и$ b.

ДокАЗАТЕЛЬСТво. Проверим сначала равенство (7). Воспользуемся формулой Стирлинга

$$
k !=\sqrt{2 \pi k}\left(\frac{k}{e}\right)^{k}\left(1+O\left(\frac{1}{k}\right)\right)
$$

для вычисления биномиального коэфффициента $C_{q}^{q_{1}\left(q_{2}+m\right)}$ :

$$
\begin{aligned}
C_{q}^{q_{1}\left(q_{2}+m\right)} & =\frac{\left(2 q_{1} q_{2}\right) !}{\left[q_{1}\left(q_{2}+m\right)\right] !\left[q_{1}\left(q_{2}-m\right)\right] !} \\
& =\sqrt{\frac{4 \pi q_{1} q_{2}}{4 \pi^{2} q_{1}^{2}\left(q_{2}^{2}-m^{2}\right)}} \cdot \frac{\left(2 q_{2}\right)^{2 q_{1} q_{2}}\left(1+O\left(z^{-2}\right)\right)}{\left(q_{2}+m\right)^{q_{1}\left(q_{2}+m\right)}\left(q_{2}-m\right)^{q_{1}\left(q_{2}-m\right)}} .
\end{aligned}
$$

Отсюда

$$
\frac{q_{1}}{2^{q}} C_{q}^{q_{1}\left(q_{2}+m\right)}=\sqrt{t}\left(1+\frac{m}{q_{2}}\right)^{-q_{1}\left(q_{2}+m\right)}\left(1-\frac{m}{q_{2}}\right)^{-q_{1}\left(q_{2}-m\right)}\left(1+O\left(\frac{M^{2}}{z^{2}}\right)\right) .
$$

Далее, замечая, что

$$
\begin{aligned}
(1 & \left.+\frac{m}{q_{2}}\right)^{q_{1}\left(q_{2}+m\right)}\left(1-\frac{m}{q_{2}}\right)^{q_{1}\left(q_{2}-m\right)} \\
& =\exp \left(q_{1} q_{2} \ln \left(1-\frac{m^{2}}{q_{2}^{2}}\right)+q_{1} m \ln \left(1+\frac{m}{q_{2}}\right)-q_{1} m \ln \left(1-\frac{m}{q_{2}}\right)\right) \\
& =\exp \left(\frac{q_{1}}{q_{2}} m^{2}+O\left(\frac{M^{4}}{z^{2}}\right)\right)=e^{\pi t m^{2}}\left(1+O\left(\frac{M^{4}}{z^{2}}\right)\right),
\end{aligned}
$$

приходим к равенству (7).

Доказательство формулы (8) проводится похожим образом:

$$
\begin{aligned}
\left(\cos \frac{\pi n}{q_{1}}\right)^{q} & =\exp \left(q \ln \left(1-\frac{\pi^{2} n^{2}}{2 q_{1}^{2}}+O\left(\frac{N^{4}}{z^{4}}\right)\right)\right)=\exp \left(q\left(-\frac{\pi^{2} n^{2}}{2 q_{1}^{2}}+O\left(\frac{N^{4}}{z^{4}}\right)\right)\right) \\
& =\exp \left(-\frac{q_{2} \pi^{2} n^{2}}{q_{1}}+O\left(\frac{N^{4}}{z^{2}}\right)\right)=e^{-\pi n^{2} / t}\left(1+O\left(\frac{N^{4}}{z^{2}}\right)\right) .
\end{aligned}
$$


ЛЕМма 3. Пусть, как и в лемме 2, выполняются условия

$$
t>0, \quad \pi t=\frac{a}{b}, \quad(a, b)=1, \quad z \geqslant 1, \quad q_{1}=a z, \quad q_{2}=b z, \quad q=2 q_{1} q_{2} .
$$

Будем также предполагать, что параметры $M$ и $N$ удовлетворяют неравенствам

$$
\frac{1}{t} \leqslant M \leqslant \sqrt{z}, \quad t \leqslant N \leqslant \sqrt{z}
$$

Тогда справедливы следующие оченки:

$$
\begin{gathered}
\sum_{m \geqslant M} e^{-\pi t m^{2}}=O\left(e^{-2 M}\right), \\
\sum_{n \geqslant N} e^{-\pi n^{2} / t}=O\left(e^{-2 N}\right), \\
\frac{q_{1}}{2^{q}} \sum_{m \geqslant M} C_{q}^{q_{1}\left(q_{2}+m\right)}=O\left(e^{-2 M}\right), \\
\sum_{N \leqslant n \leqslant q_{1} / 2}\left(\cos \frac{\pi n}{q_{1}}\right)^{q}=O\left(e^{-2 N}\right),
\end{gathered}
$$

где, как и раньше, константы в знаках $O$ могут зависеть от а $и$ b.

ДокАЗАТЕЛЬСТво. Проверим, что в каждой из четырех сумм слагаемые убьвают не медленнее, чем элементы геометрической прогрессии со знаменателем $1 / 2$. Из этого будет следовать, что каждую из сумм можно оценить первым (наибольшим) слагаемым.

Действительно, в первом случае

$$
\frac{e^{-\pi t(m+1)^{2}}}{e^{-\pi t m^{2}}}=e^{-\pi t(2 m+1)}<e^{-2 \pi t M} \leqslant e^{-2 \pi}<\frac{1}{2}
$$

и наибольшее слагаемое оценивается величиной

$$
e^{-\pi t M^{2}} \leqslant e^{-\pi M}=O\left(e^{-2 M}\right)
$$

Оценка (10) проверяется абсолютно аналогично.

Докажем формулу (11). Отношение соседних слагаемых вновь не превосходит 1/2:

$$
\begin{aligned}
\frac{C_{q}^{q_{1}\left(q_{2}+m+1\right)}}{C_{q}^{q_{1}\left(q_{2}+m\right)}} & =\frac{\left[q_{1}\left(q_{2}-m\right)\right] \cdots\left[q_{1}\left(q_{2}-m\right)-q_{1}+1\right]}{\left[q_{1}\left(q_{2}+m\right)+q_{1}\right] \cdots\left[q_{1}\left(q_{2}+m\right)+1\right]}<\left(\frac{q_{2}-m}{q_{2}+m}\right)^{q} \\
& <\left(1+\frac{m}{q_{2}}\right)^{-2 q_{1}}=e^{-2 q_{1} \ln \left(1+m / q_{2}\right)}<e^{-m q_{1} / q_{2}}=e^{-\pi t m}<e^{-\pi}<\frac{1}{2} .
\end{aligned}
$$

Кроме этого, первое слагаемое в левой части формулы (11) можно оценить при помощи леммы 2:

$$
\frac{q_{1}}{2^{q}} C_{q}^{q_{1}\left(q_{2}+M\right)}=O\left(e^{-\pi t M^{2}}\right)=O\left(e^{-2 M}\right) .
$$


Для проверки формулы (12) заметим сначала, что

$$
\begin{aligned}
\frac{\cos \left(\pi(n+1) / q_{1}\right)}{\cos \left(\pi n / q_{1}\right)} & =1+\frac{\cos \left(\pi(n+1) / q_{1}\right)-\cos \left(\pi n / q_{1}\right)}{\cos \left(\pi n / q_{1}\right)} \\
& <1-\frac{\left(\pi / q_{1}\right) \sin \left(\pi n / q_{1}\right)}{\cos \left(\pi n / q_{1}\right)}=1-\frac{\pi}{q_{1}} \operatorname{tg} \frac{\pi n}{q_{1}}<1-\frac{\pi^{2} n}{q_{1}^{2}}
\end{aligned}
$$

Поэтому

$$
\left(\frac{\cos \left(\pi(n+1) / q_{1}\right)}{\cos \left(\pi n / q_{1}\right)}\right)^{q}<\left(1-\frac{\pi^{2} n}{q_{1}^{2}}\right)^{q}<e^{-2 q_{2} \pi^{2} n / q_{1}}=e^{-\pi n / t}<e^{-\pi}<\frac{1}{2}
$$

Первое слагаемое в левой части равенства (12) вновь оценивается при помощи леммы 2 :

$$
\left(\cos \frac{\pi N}{q_{1}}\right)^{q}=O\left(e^{-\pi N^{2} / t}\right)=O\left(e^{-2 N}\right)
$$

ТЕОрема 2. При всех $t>0$ функиия $\theta(t)$, задаваемая рядом (4), удовлетворяет равенству (3).

ДоКАЗАТЕЛЬСТВО. Из абсолютной сходимости ряда (4) следует непрерьвность функции $\theta(t)$. Поэтому теорему достаточно доказать лишь для тех положительных $t$, для которых число $\pi t$ рационально.

Зафиксируем $t>0$ и определим целые $a$ и $b$ при помощи соотношений $\pi t=a / b$, $(a, b)=1$. Далее выберем $z \geqslant e^{\max \left(t, t^{-1}\right)}$ и положим

$$
M=N=\ln z, \quad q_{1}=a z, \quad q_{2}=b z, \quad q=2 q_{1} q_{2}
$$

Пользуясь последовательно формулами (11) и (7), находим, что

$$
\begin{aligned}
\frac{q_{1}}{2^{q}} \sum_{m=-q_{2}}^{q_{2}} C_{q}^{q_{1}\left(q_{2}+m\right)} & =\frac{q_{1}}{2^{q}} \sum_{|m|<M} C_{q}^{q_{1}\left(q_{2}+m\right)}+O\left(\frac{1}{z^{2}}\right) \\
& =\sqrt{t}\left(1+O\left(\frac{M^{4}}{z^{2}}\right)\right) \sum_{|m|<M} e^{-\pi t m^{2}}+O\left(\frac{1}{z^{2}}\right) \\
& =\sqrt{t} \sum_{|m|<M} e^{-\pi t m^{2}}+O\left(\frac{M^{4}}{z^{2}}\right) .
\end{aligned}
$$

Применяя оценку (9), приходим к соотношению

$$
\frac{q_{1}}{2^{q}} \sum_{m=-q_{2}}^{q_{2}} C_{q}^{q_{1}\left(q_{2}+m\right)}=\sqrt{t} \theta(t)+O\left(\frac{M^{4}}{z^{2}}\right) .
$$


Аналогично из формул (12) и (8) следует, что

$$
\begin{aligned}
\sum_{n=0}^{q_{1}-1}\left(\cos \frac{\pi n}{q_{1}}\right)^{q} & =\sum_{|n| \leqslant N}\left(\cos \frac{\pi n}{q_{1}}\right)^{q}+O\left(\frac{1}{z^{2}}\right) \\
& =\left(1+O\left(\frac{N^{4}}{z^{2}}\right)\right) \sum_{|n|<N} e^{-\pi n^{2} / t}+O\left(\frac{1}{z^{2}}\right) \\
& =\sum_{|n|<N} e^{-\pi n^{2} / t}+O\left(\frac{N^{4}}{z^{2}}\right) .
\end{aligned}
$$

Теперь из оценки (10) следует равенство

$$
\sum_{n=0}^{q_{1}-1}\left(\cos \frac{\pi n}{q_{1}}\right)^{q}=\theta\left(\frac{1}{t}\right)+O\left(\frac{N^{4}}{z^{2}}\right) .
$$

Подставляя формулы (13) и (14) в (6), получаем соотношение

$$
\sqrt{t} \theta(t)=\theta\left(\frac{1}{t}\right)+O\left(\frac{\ln ^{4} z}{z^{2}}\right) .
$$

Переходя к пределу при $z \rightarrow \infty$, приходим к нужному результату.

\section{СПИСОК ЦИТИРОВАННОЙ ЛИТЕРАТУРЫ}

[1] Коблиц Н. Введение в эллиптические кривые и модулярные формы. Новокузнецк: ИО НФМИ, 2000.

[2] Карацуба А. А. Основы аналитической теории чисел. М.: Наука, 1983.

[3] Архипов Г.И., Садовничий В.А., Чубариков В.Н. Лекции по математическому анализу. М.: Высшая школа, 1999.

[4] Титчмарш Е. Введение в теорию интегралов Фурье. М.-Л.: ОГИЗ, 1948.

Московский государственный университет им. М.В. Ломоносова

Поступило

E-mail : ustinov@mech.math.msu.su

29.01.2002 